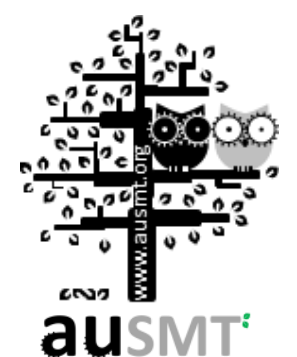

\title{
Development of a P300 Brain-Machine Interface and Design of an Elastic Mechanism for a Rehabilitation Robot
}

\author{
Han-Pang Huang1, Yi-Hung Liu², Wei-Zhi Lin', *, Zhi-Hao Kang1, \\ Ching-An Cheng1, and Tzu-Hao Huang1 \\ ${ }^{1}$ Department of Mechanical Engineering, National Taiwan University, Taiwan \\ ${ }^{2}$ Department of Mechanical Engineering, National Taipei University of technology, Taiwan \\ (Received 15 January 2014; Accepted 10 March 2014; Published on line 1 June 2015) \\ *Corresponding author email: levi5382001@gmail.com \\ DOI: $10.5875 /$ ausmt.v5i2.518
}

\begin{abstract}
This paper focuses on the development of a P300 speller and the design of a rehabilitation robot using a brain-machine interface. The combined feature set provides a norm that can be used to assess trends of the user's increased or decreased independence. The combined feature set is found to maintain a $90 \%$ sorting rate; it can also reduce the relationship of individual independence for each subject. Among the results, the highest P300 classification accuracy can be increased by $36.04 \%$. A novel adaptive coupled elastic actuator (ACEA) is proposed that uses adjustable characteristics to adapt to the applied output and input forces, thus ensuring safe human-machine interaction without the use of complex control strategies. The proposed robotic system uses variable impedance to achieve adaptability and safety in dynamic unstructured environments. This paper discusses the design, model, control, and performance of the ACEA.
\end{abstract}

Keywords: Physical human-robot interaction; coupled elastic actuation; adaptive coupled elastic actuator; compliant actuators; human-machine systems

\section{Introduction}

Brain-computer interfaces $(\mathrm{BCl})$ are a type of communication channel that does not require the use of peripheral nerves or muscles, thus transforming and directly channeling brain activity to commands for electronic devices [1], with obvious benefits for people with severe motor disabilities. Due to their high temporal resolution, electroencephalogram (EEG) signals are frequently used as inputs for $\mathrm{BCl}$ systems.

The $\mathrm{P} 300$ potential is a response to an infrequent stimulus. It usually appears in EEG signals around $300 \mathrm{~ms}$ following an infrequent stimulus. Farwell and Donchin [2, 3] proposed a P300 speller, in which the visually-evoked P300 potential is elicited by an oddball paradigm. When the user mentally focuses on a character in a spelling matrix, the $\mathrm{P} 300$ speller $\mathrm{BCl}$ system detects the targeted character and then displays the detection result on a screen, thereby enabling patients with lock-in syndrome to communicate. Previous work on P300 speller BCls used various two-class classifiers to solve the character detection problem. Hoffmann et al. [4] used boosting with orthogonal least squares as the classifier. Lenhardt et al. [5] and Bostanov [6] used the linear discriminant analysis (LDA) method to maximize the separation between classes. Based on the LDA, a Bayesian LDA classifier was further proposed in [7] and has been used in a P300 speller paradigm [8]. The support vector machine (SVM) approach has also shown satisfactory results in P300 BCI systems [9]. More recently, SVM ensembles were shown to provide better results than single SVMs in solving the problem of P300 detection [10]. The success of SVM in those P300 BCls can be attributed to the fact that it maps observations from a general set into an inner product space. and its formulation seeks to minimize structural risk [11], thus providing a better degree of generalization than traditional learning machines, such as neural networks trained by an error back-propagation algorithm 
[12].

The field of physical human-robot interaction (pHRI) focuses on the development of information interaction

Han-Pang Huang received his Ph.D. in electrical engineering from the University of Michigan, Ann Arbor, in 1986. He is a Distinguished Professor and Zhong Zhuo-Zhang Chair Professor in the Department of Mechanical Engineering and the Graduate Institute of Industrial Engineering, National Taiwan University. He is a Fellow of the CSME and the IEEE PAB, He serves as Editor of IEEE/ASME Trans. on Mechatronics, the Intl. J. of Advanced Robotics Systems, and the International Journal of Electronic Business Management. Dr. Huang's research interests include intelligent robotic systems, prosthetic hands, manufacturing automation, and control systems. Dr. Huang was a recipient of the Ford University Research Award 1996-1998; National Science Council Outstanding Research Awards, 1996-1998, 1998-2000, 2000-2002; National Science Council Distinguished Research Awards, 2002-2008; National Science Council Distinguished Research Fellow Awards, 2009, TECO Outstanding Science and Technology Research Achievement Award, 2012, and Distinguished Education Award on RFID from EPCglobal (Taiwan), 2010. He served as Guest Editor of IEEE/ASME Trans. on Mechatronics in 2001 and 2006, and of IEEE Trans. on Industrial Electronics in 2008 and 2009. He served as Editor-in-Chief of the Journal of Chinese Fuzzy System Association from September 1997 to September 1999, and of the International Journal of Fuzzy System from September 1999 to December 2002. He was Associate Editor of IEEE Trans. on Automation Science and Engineering from June 2003 to August 2005, and Board Member of the International Journal of Advanced Robotics from 2004 to 2008, and the MIT Supply ChainForum 2005-2008. He also served as the President of the Chinese Institute of Automation Engineers, Program Director of the Automation Technology Division, National Science Council; and as Adviser to the Industry Bureau, Department of Technology.

Yi-Hung Liu received his M.S. in engineering science and ocean engineering and his Ph.D. degree in mechanical engineering, both from National Taiwan University, Taiwan, in 1996 and 2003, respectively. In 2003, he joined the faculty of Chung Yuan Christian University, Taiwan, where he is currently Associate Professor of Mechanical Engineering. His research interests include machine learning, machine vision, brain-computer/robot interfaces, neuroprostheses, and intelligent system diagnosis.

Dr. Liu is a member of the IEEE and CIAE. He currently serves as Associate Editor of the International Journal of Automation and Smart Technology (AUSMT), and Guest Editor of the Journal of Neuroscience and Neuroengineering (JNSNE) issued by American Science Publishers. He is the current Co-chair of the Technical Committee on Medical Mechatronics of IEEE's SMC society. He has published 60 papers in journal and conference proceedings. He was the recipient of the 2006 Best Paper Award from the Chinese Institute of Industrial Engineers, the recipient of the Annual Best Paper Award from the 2009 Automatic Optical Inspection Forum and Competition, and the recipient of the Best Paper Award from the 2nd International Conference on Mechatronics and Applied Mechanics in 2012. He was named in Marquis' Who's Who in Asia 2007, and Who's Who in the World 2008, 2009, and 2010.

Wei-Zhi Lin received his M.D. from Chung Yuan Christian University in 2010 and is currently a Ph.D. candidate in the Department of Mechanical Engineering at National Taiwan University. His current research interests include robot hand control and design, machine learning, and pattern recognition.

Zhi-Haug Kang received his M.D. from Chung Yuan Christian University in 2010. He is currently a Ph.D. candidate in the Department of Mechanical Engineering at National Taiwan University. His current research interests include robot arm control and design, biomedical engineering, machine learning, and pattern recognition.

Ching-An Cheng received his M.S. from the Department of Mechanical Engineering at National Taiwan University in 2013. His research interests include robot control theory, machine learning, and pattern recognition.

Tzu-Hao Huang received his Ph.D. from the Department of Mechanical Engineering at National Taiwan University in 2013. His research interests include robot hand design and control, biomedical engineering, machine learning, and pattern recognition. and control systems by which humans can control robots. Such systems must make trade-offs between safety and performance [13].

Installing additional sensors on the controllers of rigid robots has been shown to effectively improve safety [14]. However, a robot's dynamic performance is limited by insufficient bandwidth. Furthermore, the natural dynamics of the system may be affected by extrinsic dynamic environmental factors.

The most critical feature of modern actuator designs is the stiffness constant of the series elastic component, a physical quantity that dominates the bandwidth and payload capacity of the overall system and the safety level of the pHRI. A system design of this type should always determine a maximum allowable output impedance and a maximum tolerable mechanism restriction on the system to define the upper bound of the stiffness constant, with the lower bound defined by a minimum acceptable large force bandwidth [15]. Stiff systems exhibit more rapid responses to load changes and have greater capacity to handle heavy objects but, with respect to the pHRI, may result in a more acute impulse shock which could lead to serious injury of people nearby. In contrast, soft systems exhibit slower responses to load changes and are less able to handle heavy objects, but their motions are explicitly designed to protect humans during manipulation. In general, a compromise between safety and performance is difficult to achieve.

The major objective of this study is to provide an inherently adjustable series elastic device for a pHRI. A novel adaptive coupled elastic actuator (ACEA) is developed, featuring characteristics which can adapt to changes in the applied output force and input force.

\section{Materials and Methods}

\section{Data Acquisition and Preprocessing}

Our system uses six monochannels, each receiving EEG signals from a distinct electrode, and individually placed on $\mathrm{Fz}, \mathrm{Fcz}, \mathrm{Cz}, \mathrm{Pz}, \mathrm{P7}$, and $\mathrm{P} 8$ in a $10-20$ system. The potential measured from each of the first four $(F z, F c z, C z$, $\mathrm{Pz}$ ) is further subtracted from the average of $\mathrm{P} 7$ and $\mathrm{P} 8$ potentials for each of four individual signals. At the preprocessing stage, $0.1-70 \mathrm{~Hz}$ band-pass filtering is performed. The acquired EEG signals are amplified with a gain of 24,000. The signals are then digitized and sent to a computer via the DAQ device (National Instrument Corp., USA). The received digitized EEG signals are called EEG data.

EEG data collection is performed at a sampling rate of $250 \mathrm{~Hz}$, using software developed by the authors in $\mathrm{C \#}$. The software first presents a $6 \times 6$ spelling matrix (Figure 
1) with row or column intensification (stimulus).

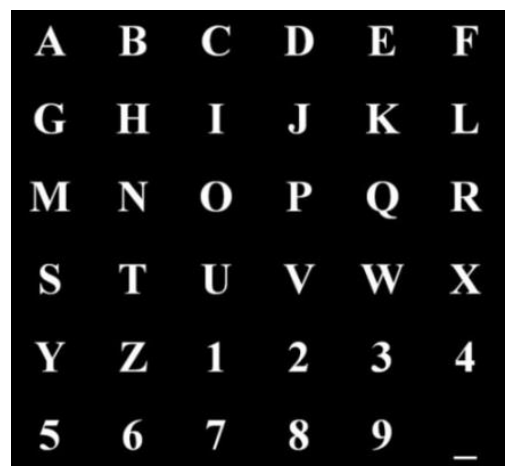

Figure 1. P300 speller.

When a stimulus is presented, the corresponding stimulus code is embedded in the data stream in a timelocked manner. Through the stimulus code, the first 500 ms data (i.e., one epoch) from each channel can be automatically obtained. That is, when a particular row or column is intensified (flashed), we get the vector

$$
\boldsymbol{x}=\left[x_{1}(1), \ldots, x_{1}(N), x_{2}(N), x_{K}(1), \ldots, x_{K}(N)\right]^{T}
$$

where $\mathrm{k}$ and $\mathrm{N}$ respectively denote the number of channels and the number of samples, and $\mathrm{x} \_\mathrm{i}(\mathrm{j})$ denotes the jth sample from the ith channel. Here, $\mathrm{N}=125$ and $\mathrm{K}$ $=4$.

\section{Data Collection}

Adapting the approach proposed in [16] out data collection procedure contains two stages: 1 ) preparation and 2) dataset collection.

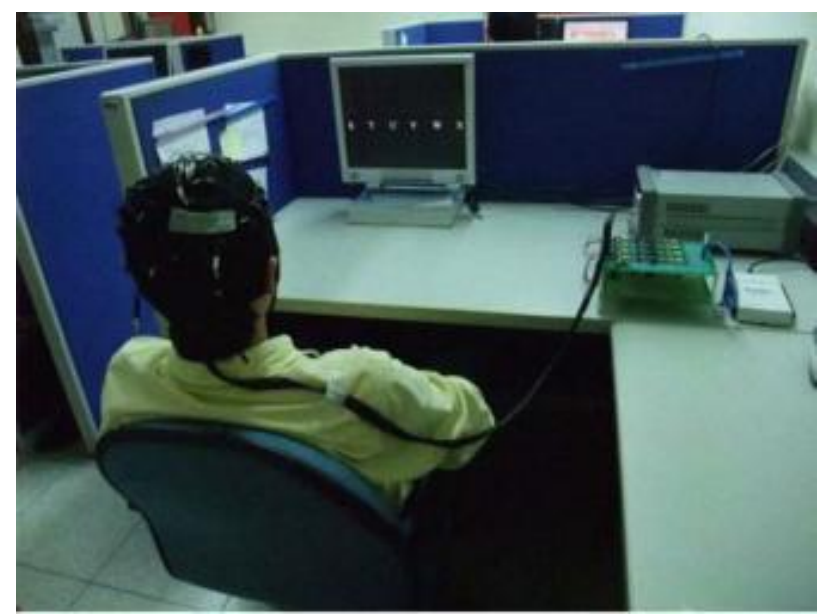

Figure 2. During data collection the computer is placed in front the blue cubicle wall.

\section{Preparation}

During the preparation stage, an electro-cap is attached to the subject's head, using electrolyte gel to reduce impedance. The subject sits about $90 \mathrm{~cm}$ in front of a 17-inch LCD display (see Figure 2).

\section{Dataset Collection}

During the training stage, the subject is asked to focus on a sequence of single characters provided by the experimenter. First, one of the characters is illuminated on the LCD for $4 \mathrm{~s}$, during which it is visible to the subject. After the attention-catching intensification, a 2.5-s preparation period is given before the data collection procedure. As the subject focuses his/her attention on the target character, the system initiates a 10-round intensification process where presenting 12 randomly ordered visual stimuli in each round. The rows and columns of the matrix are intensified successively at random for $100 \mathrm{~ms}$; after a 75 -ms inter-stimuli interval, the next stimulus is presented. Each round presents 10 individual stimuli, with a pause of $0.5 \mathrm{~s}$ between rounds. Therefore, a 10-round intensification process will take $27.75 \mathrm{~s}$.

In each round, the presentation of a particular stimulus produces a corresponding vector. Following Eq. (1) for the intensification of the ith row in the $j$ th round produces a column vector $x_{i j} \in R^{N \times K}$. Following five rounds, we obtain the average vector associated with the ith row, where $A$ is the number of rounds. Note that such averaged vectors (referred to as "patterns") are used as the classifier input. Also, if this intensified row contains the target character, then $x_{i}$ contains a P300 potential and is used as a positive (target) training pattern; if not, it is used as a negative training pattern. The completed training produces a training set containing $n_{p}$ positive (P300) patterns and $n_{n}$ negative (non-P300) patterns.

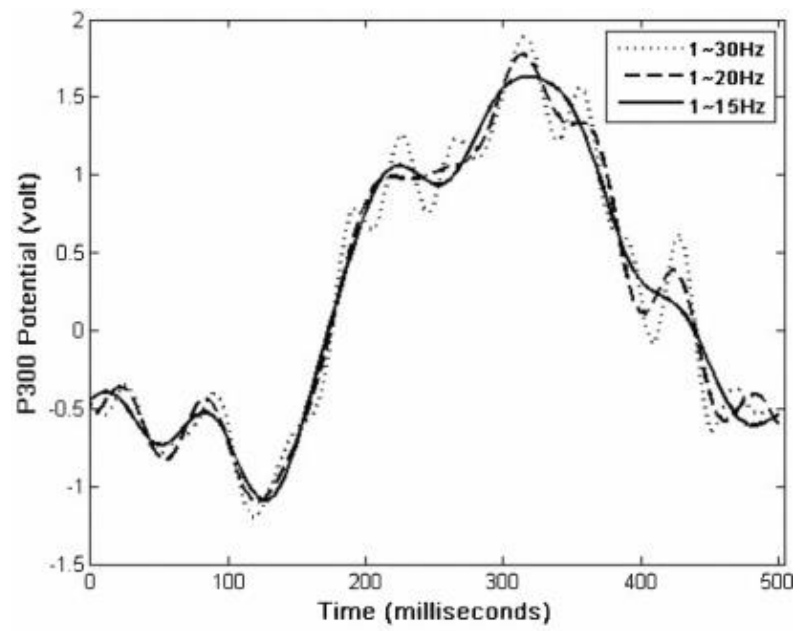

Figure 3. Band-pass filtered P300 potentials using inverse DFT. The $x$-axis is the time from the stimulus presentation.

Figure 3 shows P300 potentials recorded from Pz. We found that the SVM achieves the best classification accuracy when the frequency band is set at 1-15 $\mathrm{Hz}$. 
Within this band, the signal to noise ratio (SNR) of the EEG signals is greatly increased due to the complete elimination of $60-\mathrm{Hz}$ line noise and the significantly reduced impact of electromyography signals $(10-400 \mathrm{~Hz}$ [17]). However, such reconstruction cannot remove electrooculography (EOG) signals since they lie in low frequencies below $10 \mathrm{~Hz}$ [18]. To avoid ocular artifacts, the subjects were asked to avoid eye movement during the experiments.

\section{Feature Extraction and Classification}

Feature extraction can be divided into two parts : 1) the EEG signal superposition of the average after feature extraction (there are three features of the area: N100 and P300 time and slope); 2) the EEG signal is depicted in a binary image and turned into a closed curve using the boundary extraction algorithm [19]. The edge of the binary image is recorded by Fourier descriptors. The edge information is then transformed into the form of frequency data and we can obtain the edge of the center $\left(\mathrm{x}_{\text {center }}, \mathrm{y}_{\text {center }}\right)$. Each feature is described in Figure 4.

\section{(a) Area}

The area is defined as the sum of all EEG signals between 200 and $500 \mathrm{~ms}$.

\section{(b) Time of N100 and P300}

N100 time is the local trough of the signal between 50 and 170 ms and P300 time is the local peak of the signal between 220 and $500 \mathrm{~ms}$.

\section{(c) Slope}

$$
m=\frac{\text { P300volt }- \text { N100volt }}{\text { P300time }- \text { N100time }}
$$

where $P 300$ volt is the $P 300$ peak value N100volt is the N100 trough value P300time is the P300 time N100time is the N100 time.

\section{(d) Fourier Descriptor (FD) [20] and Center}

Assume that the edges of an image can make use of the Cartesian coordinate system to obtain the following coordinates on $(x[m], y[m]), m=1,2, \ldots, L$ ( $L$ is the total number of image edges). From these coordinates on $(x[m]$, $y[m])$, Eqs. (3) and (4) can obtain the Fourier coefficient $(a[n], b[n])$

$$
\begin{aligned}
& a[n]=\frac{1}{L} \sum_{m=1}^{L} x[m] e^{-j n \omega_{0} m} \\
& b[n]=\frac{1}{L} \sum_{m=1}^{L} y[m] e^{-j n \omega_{0} m}
\end{aligned}
$$

From Eq. (5) we obtain get the Fourier descriptor $s[n]$

$$
\begin{aligned}
& s[n]=\frac{r[n]}{r[1]} \\
& r[n]=\sqrt{|a[n]|^{2}+|b[n]|^{2}} .
\end{aligned}
$$

Equation (6) gives the center of the edge ( $\left.x_{\text {center }}, y_{\text {center }}\right)$

$$
x_{\text {center }}=\frac{\sum_{m=1}^{L} x[m]}{L}, \mathrm{y}_{\text {center }}=\frac{\sum_{m=1}^{L} y[m]}{L} .
$$

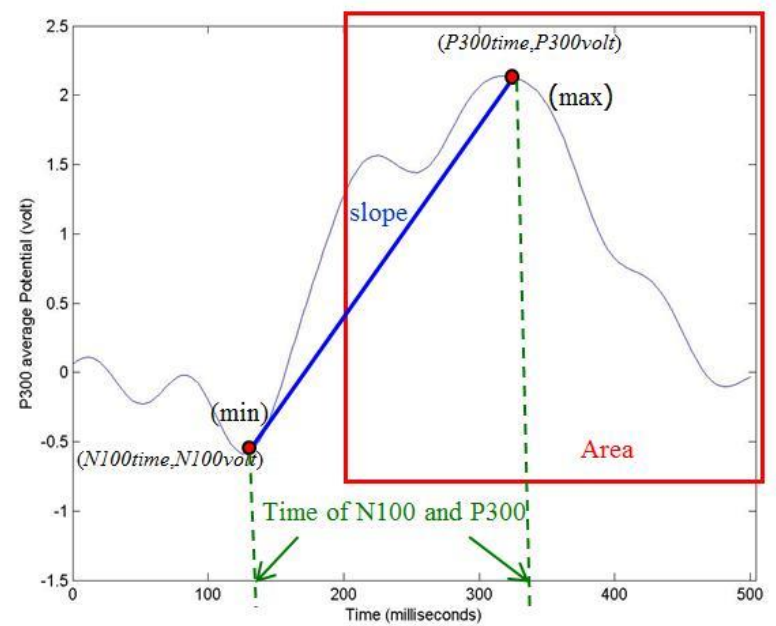

Figure 4. Description of the different features.

SVM is used as a post-extraction classifier. In the SVM, the training set is given as $\left\{x_{i}, y_{i}\right\}_{i=1}^{n}$, where $\mathbf{x}_{i} \in R^{d}$ are the training patterns and $y_{i} \in\{-1,+1\}$ are the class labels. Here, $d=500$. Let $w$ and $b$ respectively be the weight vector and the bias of the separating hyperplane. The objective of the SVM is to find the optimal hyperplane by maximizing the margin of separation and minimizing the training errors, formulated as the optimization problem.

$$
\begin{array}{cc}
\min _{f, b, \xi} \frac{1}{2}\|w\|^{2}+C \sum_{i}^{N} \xi_{i}, & \text { for } i=1,2, \ldots \ldots, N, \\
\text { s.t. } y_{i}\left(w^{T} \phi\left(x_{i}\right)+b\right) \geq 1-\xi_{i}, & \forall \mathrm{i}, \\
\xi_{i} \geq 0, & \forall \mathrm{i},
\end{array}
$$

The dual problem of the SVM is as follows:

$$
\begin{array}{ll}
\max _{f, b, \xi}-\frac{1}{2} \sum_{i}^{N} \alpha_{i} \alpha_{j} y_{i} y_{j} k\left(x_{i}, x_{j}\right)+\sum_{i}^{N} \alpha_{i}, \text { for } i=1,2, \ldots \ldots, N, & \\
\text { s.t. , } \sum_{i}^{N} \alpha_{i} y_{i}, & \forall \mathrm{i}, \\
0 \leq \xi_{i} \leq C, &
\end{array}
$$


Finally, we can obtain the decision function

$$
f(x)=\sum_{i}^{N} \alpha_{i}^{*} K\left(x, x_{i}\right)+b^{*}
$$

The kernel type is chosen as the Gaussian kernel

$$
K_{\text {Gaussian }}\left(x_{i}, x_{j}\right)=\phi\left(x_{i}\right) \phi\left(x_{j}\right)^{T}=\exp \left(\frac{\left\|x_{i}-x_{j}\right\|_{2}^{2}}{2 \sigma^{2}}\right)
$$

Two-fold cross-validation was run 10 times to evaluate the classification rate. The SVM determines the most probable row and column by the following two decision functions:

$$
\begin{aligned}
& r^{*}=\arg \max _{r=1, \ldots, n_{r}}\left\{\sum_{i=1}^{n} \alpha_{i} y_{i} k\left(x_{\text {row }}^{(r)}, x_{i}\right)+b\right\} \\
& c^{*}=\arg \max _{c=1, \ldots, n_{c}}\left\{\sum_{i=1}^{n} \alpha_{i} y_{i} k\left(x_{\text {col }}^{(c)}, x_{i}\right)+b\right\}
\end{aligned}
$$

where $n_{r}$ and $n_{c}$ are respectively the row and column numbers in the character matrix $\left(n_{r}=n_{c}=6\right)$, and $\alpha_{i}$ are Lagrange multipliers. Once the indices are determined, the character is recognized.

\section{Rehabilitation System}

\section{Design Concept of the ACEA}

Using a set of two different elastic components (one with soft stiffness and the other with hard stiffness) may alleviate the aforementioned drawbacks of traditional series elastic actuator (SEA) systems. A new approach ACEA is proposed, and the model is shown in Figure, where $M_{1}, X_{l}$, and $F_{l}$ respectively denote the link mass, displacement of the output link and force on the link; $M_{m}, X_{m}$ and $F_{m}$ respectively denote the actuator mass, displacement of the actuator and input force of the actuator; $K_{s}$ and $K_{h}$ respectively denote the stiffness of the soft and hard elastic elements.

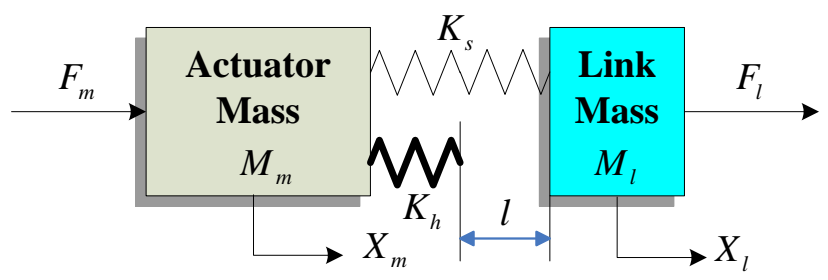

Figure 5. Model of proposed adaptive coupled elastic actuator.
$\Delta X_{s}=\left(X_{m}-X_{l}\right)$ is always positive or equal to zero, and by defining the critical length $I\left(t, \Delta X_{s}\right)$ as the difference in displacement between the positions of the hard elastic element and the output link at time $t$, the equivalent transmission stiffness of the ACEA approach can be shown as

$$
K_{t}=\left\{\begin{array}{c}
K_{s}, \text { if } F_{l} \leq F_{t h} \text { or } \mathrm{I}\left(t, \Delta X_{s}\right)>0 \\
K_{h}+K_{s}, \text { otherwise }
\end{array}\right.
$$

where $F_{t h}=K_{s} \cdot I(t, 0)$ is the threshold force that equals $K_{s}$ multiplied by the preset critical length $I(t, 0)$ associated with $\Delta X_{s}=0$, and $F_{l}=K_{s} \cdot \Delta X_{s}$ is the restored force provided by the deflection when the soft elastic element is stressed. The critical length $l$ that should be adaptive to the applied output force and input force determines the inherent stiffness of the actuation approach, meaning that the system is capable of dividing the total input force into direct driving and stiffness shifting forces, two forces that both contribute to the output force. Thus, the ACEA can divide the torque generation into separate low- and high-frequency parallel actuators by adjusting $I$ adaptively, as shown in Figure .



Figure 6. Adaptive coupled elastic actuator concept, partitioning torque into low- and high-frequency components.

By varying an adjustable $I$ accompanied by proper active control, the actuator may obtain any desired threshold force/torque adaptive to task-oriented strategies. Compared to previous compliant or stiff actuators, such as SEA, actuators using the proposed ACEA approach exhibit the desired intrinsic lower or higher output impedance. The relationship between an external load and deflection can be seen in Figure, and the approximate proposed operation states of an ACEA system can be seen in Table 1.

By assuming that the total compression distance 


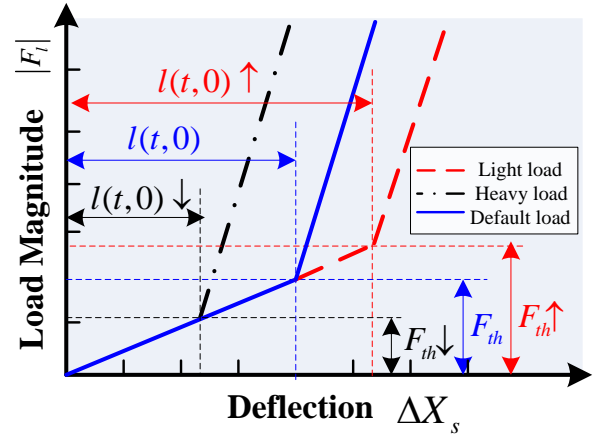

Figure 7. Properties of adaptive coupled elastic actuator; the relationship between load and deflection.

Table 1. Different proposed operation states of an ACEA system.

\begin{tabular}{ccc}
\hline $\begin{array}{c}\text { Operation } \\
\text { speed }\end{array}$ & $\begin{array}{c}\text { Hard elastic } \\
\text { element }\end{array}$ & $\begin{array}{c}\text { Soft elastic } \\
\text { element }\end{array}$ \\
\hline High & Performance & Interaction \\
Low & $\begin{array}{c}\text { Performance } \\
\text { and Interaction }\end{array}$ & Interaction \\
\hline
\end{tabular}

Mechanism Design

The critical issues of the ACEA system are how to design a mechanism and a control system to determine and adjust the stiffness of the soft and hard elastic components in advance. As shown in Fig. 8, utilizing only one actuator, the ACEA actuator, is designed to provide a favorable solution to adjust the critical length by a torque switch mechanism.

In this design, a worm drives a worm gear through two sets of preloaded soft linear compression springs, initially restraining the movement of the worm shaft in its axial direction. One set of hard linear compression springs will restrain the movement of the worm shaft in its axial direction as the critical length falls to zero.

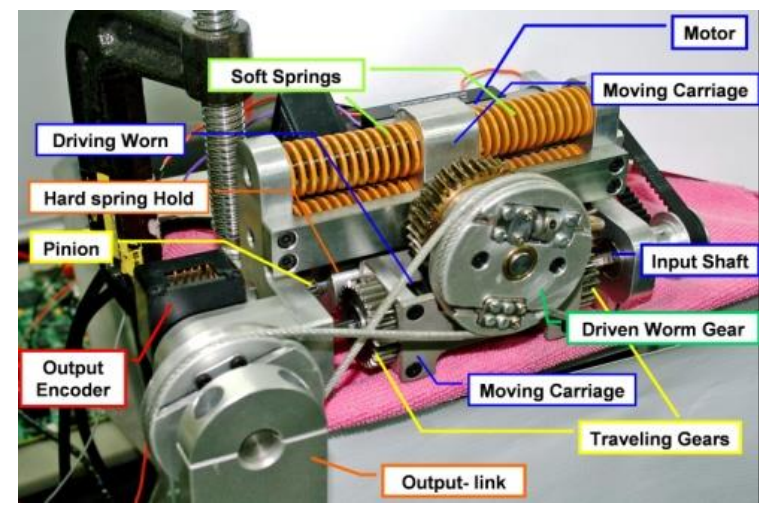

Figure 8. One-DOF adaptive coupled elastic actuator.

Specifically, the torque switch mechanism consists of a pair of traveling gears and corresponding pinions to drive a both-end-thread screw that can simultaneously convey two movable hard spring holders along the screw. This mechanism can channel and switch the input torque into either direct output torque or input torque to adjust the critical length, permitting the ACEA to vary according to the load torque and the input torque. The ACEA working principle is demonstrated in Figure, where $F_{a d}$ is the critical length adjusting force, $F_{e n}$ is the environment force, $L_{a c}$ is the displacement between the no-load position and the actuation position in which the torque switch mechanism starts to work, $\tau_{m}$ is input actuating motor torque, and $\tau_{e n}$ is environmental torque.

In Fig. 9(a), increasing the load torque on the output shaft makes the driving worm move along the input shaft where a motor provides the input force. Meanwhile, the critical length is gradually shortened. The traveling gear then moves with the worm along the input shaft where it encounters the corresponding pinion that drives the screw if the increasing load torque exceeds the threshold torque (see Fig. 9(b)). The engagement of the traveling gear and the pinion causes two movable hard spring holders (the critical length adjusters) to move along the screw, and either shorten or extend the critical length based on the direction of the input torque (see Fig. 9(c)) until the engagement of these two gears is relieved and no more action will be taken to change $l$ and $l(t, 0)$ (see Fig. $9(d))$. Therefore, the newly specified $l^{\prime}$ and $l^{\prime}(t, 0)$ will be defined (see Fig. 9(e)).

Through the torque switch mechanism, the system can mechanically alter the critical length/force in response to a light load within the first range of handling a relatively light load, within the first range of quick operation to make small output impedance responses, or in response to a heavy load within the second range of handling a relatively heavy load or the second range of slowly operating to make large output impedance responses.

\section{Experimental Results}

\section{P300 Experiment Results}

First, we discuss the relationship between the numbers of Fourier descriptors and accuracy. As seen in Fig. 10, increasing the number of descriptors increases the accuracy level. However, no further improvements are made with more than four, but each additional descriptor increases computational complexity. Therefore, we took the first four Fourier descriptors (FD4) as a feature. 


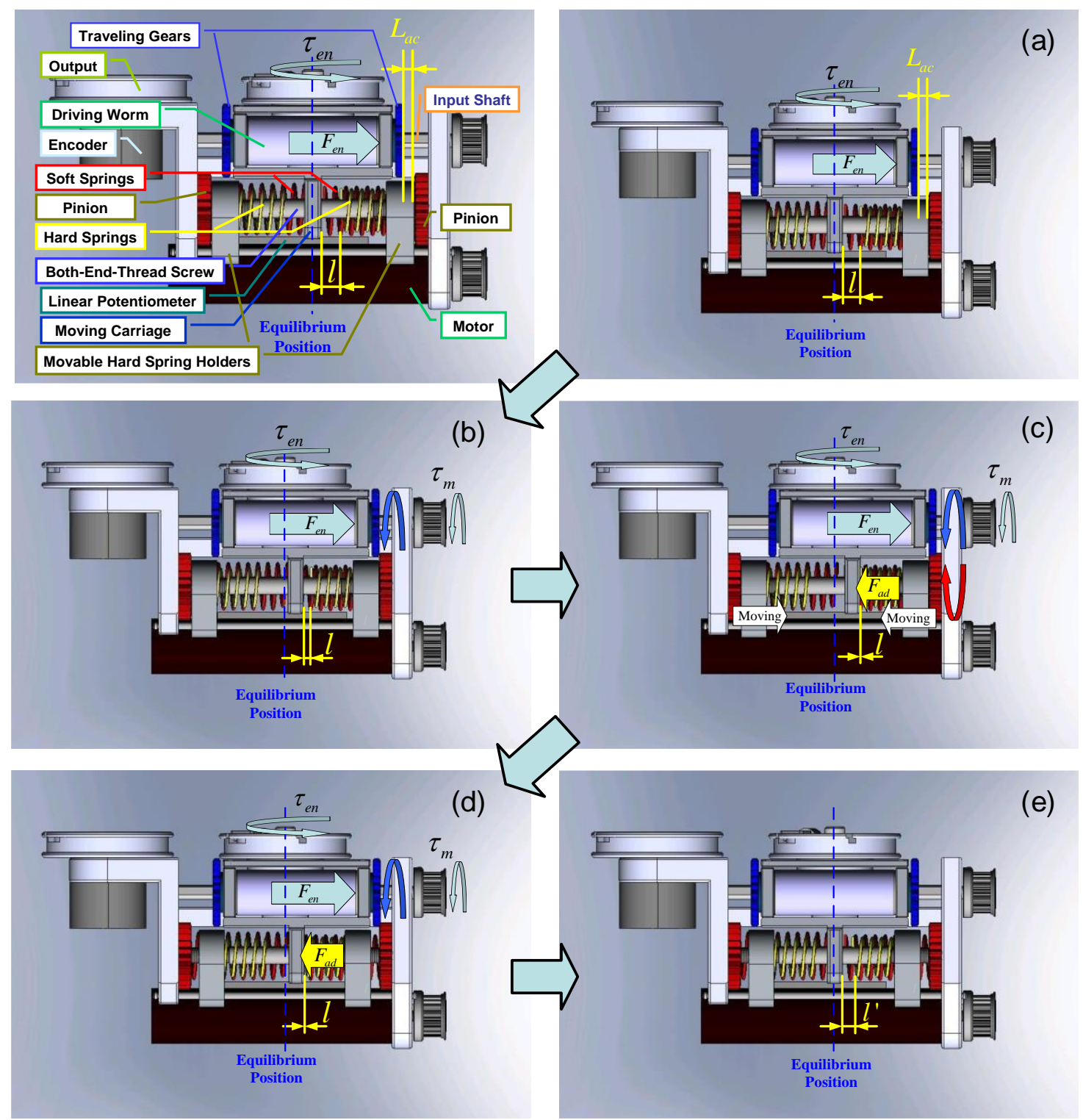

Figure 9. Torque switch operation process (view from bottom to top).

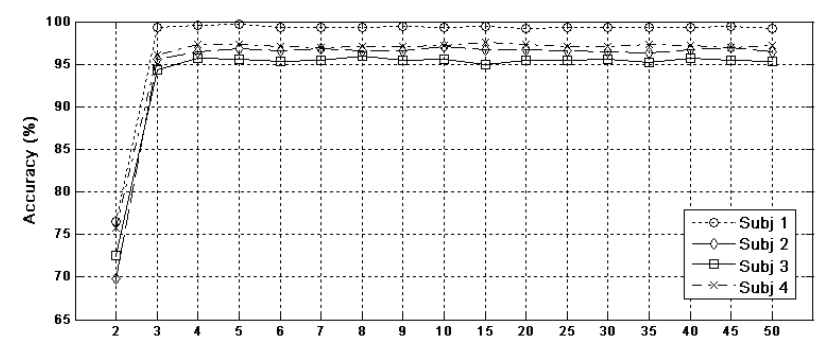

Figure 10. Relationship between different Fourier descriptors and classification rate.

In the experimental results, $A, S, M, C$ and $F$ respectively stand for area, slope, time of P300 and N100, center and Fourier descriptor. Raw represents the original data and the training time is presented in parenthesis.

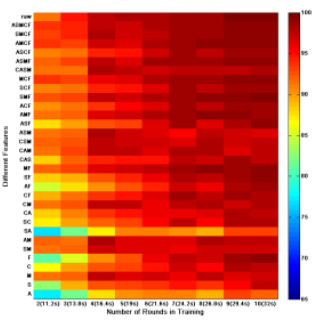

Subj1

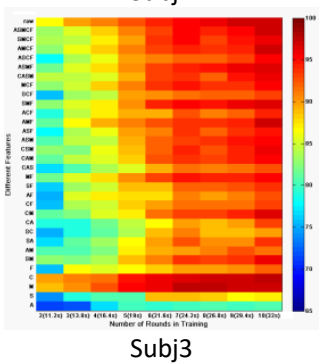

Figure 11. Different feature extraction combination of classification results.

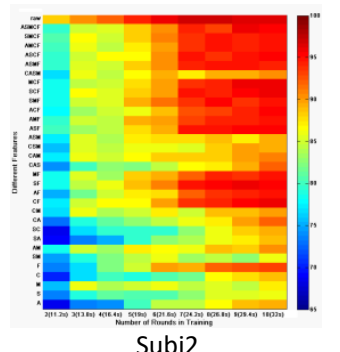

Subj2

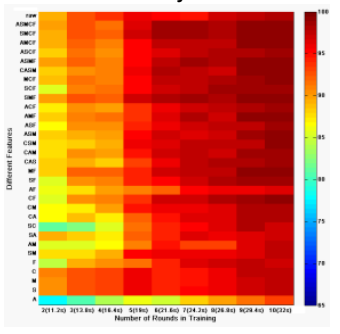

Subj4 
In Fig. 11, first we consider the five individual features, $A, S, M, C$ and $F$. $M$ and $F$ produce better results, while $A$ produces the worst result. Increasing the number of feature combinations increases the accuracy results, and the combination ASMCF can produce a level of accuracy similar to that of the raw data, while the SMCF feature combination approximates the classification rate of the raw data. In addition, including feature $F$ significantly improves accuracy results in Subj2. Different combinations of features produce different results and have different effects on different subjects. Therefore we chose the combination of features that produces best results for all four subjects to explore the relationship for EEG signal individual independence of users.

Table 2 shows data trained using the SVM model for different subjects and their corresponding training data. As shown along the diagonal, accuracy results are not high.

Table 2. Raw data of individual independence test: results of accuracy (\%).

\begin{tabular}{ccccc}
\hline Subj & 1 & 2 & 3 & 4 \\
\hline 1 & 98.78 & 64.02 & 62.80 & 68.90 \\
2 & 62.32 & 95.12 & 71.95 & 82.32 \\
3 & 72.07 & 81.10 & 98.78 & 75.73 \\
4 & 75.12 & 65.37 & 67.80 & 96.34 \\
\hline
\end{tabular}

Therefore, we hope that feature extraction will improve the classification rate outside the diagonal. Better results from Fig. 11 (specifically SF, MF, AMF, ASM, SMF, SCF, SMCF, and ASMCF) were selected for the individual independence test. The results are shown in

Table 3 to Table 10. A comparison formula is used to calculate the accuracy of the raw data after feature extraction. The change in accuracy is determined by Eq. (14).

$$
Z=\frac{Y-X}{X}
$$

$X$ and $Y$ are respectively the original signal and the post-feature extraction classification of the individual independence test and $Z$ is the calculated result. A negative value of $Z$ indicates represents this particular feature combination does not reduce the independence between individuals, while positive values indicate the opposite.

Table 3. SF features of the individual independence test results for accuracy (\%).

\begin{tabular}{ccccc}
\hline Subj & 1 & 2 & 3 & 4 \\
\hline 1 & $95.12(-3.70)$ & $80.80(26.21)$ & $81.10(29.13)$ & $90.24(30.97)$ \\
2 & $75.12(20.55)$ & $92.85(-2.38)$ & $81.71(13.56)$ & $90.07(9.42)$ \\
3 & $68.41(-5.08)$ & $82.31(1.50)$ & $92.02(-6.84)$ & $73.90(-2.42)$ \\
4 & $71.46(-4.87)$ & $62.32(-4.66)$ & $67.80(0)$ & $95.12(-1.27)$ \\
\hline
\end{tabular}

Table 4. MF features of the individual independence test results for

\begin{tabular}{|c|c|c|c|c|}
\hline Subj & 1 & 2 & 3 & 4 \\
\hline 1 & $97.56(-1.23)$ & $83.54(30.48)$ & $81.71(30.10)$ & $90.12(30.80)$ \\
\hline 2 & $75.12(20.55)$ & $92.24(-3.03)$ & $83.54(16.10)$ & $90.07(9.42)$ \\
\hline 3 & $75.12(4.23)$ & $81.10(0)$ & $92.07(-6.79)$ & $74.51(-1.64)$ \\
\hline 4 & $75.12(0)$ & $62.93(-3.73)$ & $75.12(10.79)$ & $95.12(-1.27)$ \\
\hline
\end{tabular}

Table 5. AMF features of the individual independence test results for accuracy (\%).

\begin{tabular}{ccccc}
\hline Subj & 1 & 2 & 3 & 4 \\
\hline 1 & $98.78(0)$ & $82.32(28.57)$ & $82.93(32.04)$ & $90.85(31.86)$ \\
2 & $73.29(17.61)$ & $93.46(-1.74)$ & $84.76(17.80)$ & $91.29(10.90)$ \\
3 & $72.07(0)$ & $82.32(1.50)$ & $94.51(-8.64)$ & $73.90(-3.22)$ \\
4 & $75.12(0)$ & $62.93(-3.73)$ & $66.59(-0.90)$ & $95.73(-0.63)$ \\
\hline
\end{tabular}

Table 6. ASM features of the individual independence test results for accuracy (\%)

\begin{tabular}{ccccc}
\hline Subj & 1 & 2 & 3 & 4 \\
\hline 1 & $95.73(-3.09)$ & $83.54(30.48)$ & $83.54(33.01)$ & $92.51(34.27)$ \\
2 & $75.12(20.55)$ & $94.51(-0.64)$ & $85.37(18.64)$ & $88.00(6.90)$ \\
3 & $75.12(4.23)$ & $82.32(1.50)$ & $90.24(-8.64)$ & $73.29(-3.22)$ \\
\hline 4 & $76.34(1.62)$ & $62.93(-3.73)$ & $67.20(-0.9)$ & $95.73(-0.63)$ \\
\hline
\end{tabular}

Table 7. SMF features of the individual independence test results for accuracy (\%)

\begin{tabular}{ccccc}
\hline Subj & 1 & 2 & 3 & 4 \\
\hline 1 & $98.78(0)$ & $84.76(32.38)$ & $82.32(31.07)$ & $92.51(34.27)$ \\
2 & $74.51(19.57)$ & $93.46(-1.74)$ & $82.32(14.41)$ & $91.29(10.90)$ \\
3 & $75.12(4.23)$ & $83.54(3.01)$ & $90.85(-8.02)$ & $76.34(0.81)$ \\
\hline 4 & $75.73(0.81)$ & $63.54(-2.80)$ & $69.02(1.80)$ & $97.56(1.27)$ \\
\hline
\end{tabular}

Table 8. SCF features of the individual independence test results for accuracy (\%).

\begin{tabular}{ccccc}
\hline Subj & 1 & 2 & 3 & 4 \\
\hline 1 & $96.95(-1.85)$ & $85.37(33.33)$ & $80.49(28.16)$ & $89.63(30.09)$ \\
2 & $75.12(20.55)$ & $91.46(-3.85)$ & $82.93(15.25)$ & $91.44(11.08)$ \\
3 & $79.02(9.64)$ & $82.32(1.50)$ & $89.02(-9.88)$ & $75.73(0)$ \\
4 & $74.51(-0.81)$ & $62.32(-4.66)$ & $67.80(0)$ & $95.12(-1.27)$ \\
\hline
\end{tabular}

Table 9. SMCF features of the individual independence test results accuracy (\%)

\begin{tabular}{ccccc}
\hline Subj & 1 & 2 & 3 & 4 \\
\hline 1 & $98.78(0)$ & $84.76(32.38)$ & $84.15(33.98)$ & $93.73(36.04)$ \\
2 & $75.73(21.53)$ & $92.46(-2.79)$ & $82.93(15.25)$ & $90.02(9.36)$ \\
3 & $75.12(4.23)$ & $84.76(4.51)$ & $91.46(-7.41)$ & $75.73(0)$ \\
4 & $75.64(0.69)$ & $61.71(-5.6)$ & $78.41(15.65)$ & $98.78(2.53)$ \\
\hline
\end{tabular}

Table 10. ASMCF features of the individual independence test results for accuracy (\%).

\begin{tabular}{ccccc}
\hline Subj & 1 & 2 & 3 & 4 \\
\hline 1 & $100.00(1.23)$ & $83.54(30.48)$ & $78.66(25.24)$ & $90.24(30.97)$ \\
\hline 2 & $75.12(20.55)$ & $92.68(-2.56)$ & $82.93(15.25)$ & $92.07(11.85)$ \\
\hline 3 & $73.29(1.69)$ & $84.15(3.76)$ & $92.68(-6.71)$ & $75.12(-0 . .81)$ \\
\hline 4 & $74.51(-0.81)$ & $62.32(-4.66)$ & $69.02(1.80)$ & $98.78(2.53)$ \\
\hline
\end{tabular}

In Tables 3 to 10, the rows show the training data of different subjects while the columns show the test data of different subjects. The value in parentheses indicates whether the feature extraction rose or declined in terms of individual independence. If positive, the result can reduce the subjects' individual independence. The experimental results show that some feature extractions can reduce individual independence. The SMCF for reducing the effect of individual independence (Table 8) 
shows the greatest increase of $36.04 \%$, thus improving accuracy from $68.9 \%$ to $93.73 \%$. We get the feature combination of the SMCF which is done five times superposition; the accuracy all subjects can be maintained at about $90 \%$ while the individual independence declines.

\section{Human-Robot Interaction}

The dynamics of a robotic system can be drastically changed through interaction with a human operator since the motion of the human is too complicated to be modeled or predicted.

We chose the control mode of the designed system to match different operating situations according to whether or not the robot was interacting with a human operator. In the beginning, the robot was set to track the given output link trajectory under the output link angle control without any interaction with a human subject or the environment. The human operator then tried to randomly guide the output link under the motor angle control or zero impedance control.

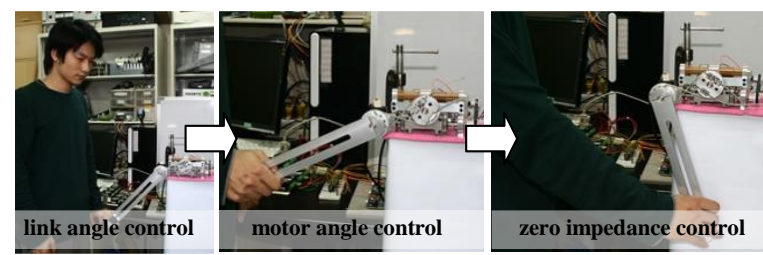

(a) Human-robot interaction experiment with a human operator

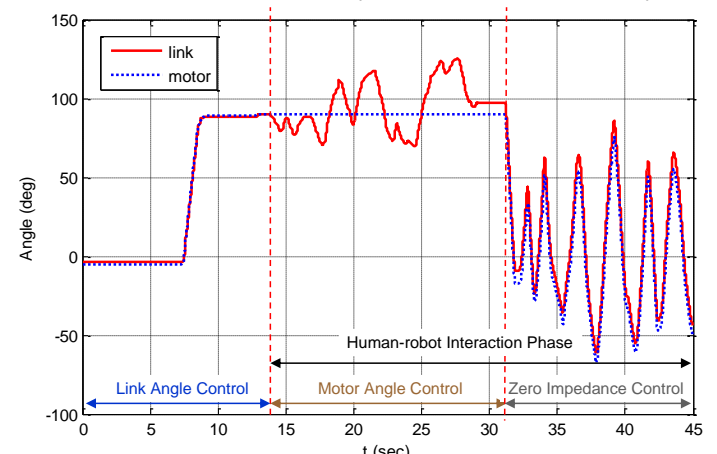

(b) Link angle and motor angle under different control modes Figure 12. Human-robot interaction experiment under three control modes.

As shown in Fig. 12, the experimental results demonstrated that the robot can track the given output link trajectory quite using the output link angle control despite the effect of the link dynamics as the main resistive torque source. Because of the joint flexibility, the human operator can guide the robot's movement. However, the operator could easily and randomly guide the robot under zero impedance control where the desired output torque was set at zero, despite some relatively small resistive torque observed in the experiment.

\section{Conclusions}

This paper focused on the development of a P300 speller and design of a rehabilitation robot for brainmachine interaction. The extracted feature sets were validated by individual independence. The results showed that the combination of the extracted feature sets and SMCF maintains a $90 \%$ classification rate and reduces the effective individual independence. By using the P300 speller and reducing the effective individual independence with P300 event related potential (ERP), this technique can provide people with physical disabilities with increased convenience. The proposed adaptive coupled elastic actuator allows a rehabilitation robot system to use variable impedance to achieve adaptability and safety in dynamic unstructured environments.

Future work will focus on the development and testing of an online brain-controlled rehabilitation robot, featuring reduced detection time and increased safety.

\section{Acknowledgments}

This work was partially supported by the National Science Council of the Republic of China (R.O.C.) under grants NSC 100-2221-E-002-12 7-MY3 and NSC 100-2221E-002-077-MY3.

\section{References}

[1] N. Birbaumer, J. R. Wolpaw, D. J. McFarland, G. Pfurtscheller, and T. M. Vaughan, "Braincomputer interfaces for communication and control," Clinical Neurophysiology, vol. 113, no. 5, pp. 767-791, 2002. doi: $10.1145 / 1941487.1941506$

[2] L. A. Farwell and E. Donchin, "Talking off the top of your head: Toward a mental prosthesis utilizing event-related brain potentials," Electroencephalography and Clinical Neurophysiology, vol. 70, no. 6, pp. 510-523, 1988. doi: 10.1016/0013-4694(88)90149-6

[3] E. Donchin, K. M. Spencer, and R. Wijesinghe, "The mental prosthesis: Assessing the speed of a p300based brain-computer interface," IEEE Transactions on Rehabilitation Engineering, vol. 8, no. 2, pp. 174179, 2000. doi: $10.1109 / 86.847808$

[4] U. Hoffmann, G. Garcia, J. Vesin, K. Diserens, and T. Ebrahimi, "A boosting approach to p300 detection with application to brain-computer interfaces," in proceeding of International IEEE EMBS Conference on Neural Engineering, Arlington, USA, March 16-19, 2005, pp. 97-100. 
doi: $\underline{10.1109 / C N E .2005 .1419562}$

[5] A. Lenhardt, M. Kaper, and H. J. Ritter, "An adaptive p300-based online brain-computer interface," IEEE Transactions on Neural Systems and Rehabilitation Engineering, vol. 16, no. 2, pp. 121-130, 2008. doi: 10.1109/TNSRE.2007.912816

[6] V. Bostanov, "Bci competition 2003-data sets ib and iib: Feature extraction from event-related brain potentials with the continuous wavelet transform and the t-value scalogram," IEEE Transactions on Biomedical Engineering, vol. 51, no. 6, pp. 10571061, 2004.

doi: $10.1109 / T B M E .2004 .826702$

[7] U. Hoffmann, J. M. Vesin, T. Ebrahimi, and K. Diserens, "An efficient p300-based braincomputer interface for disabled subjects," Journal of Neuroscience Methods, vol. 167, no. 1, pp. 115125, 2008.

doi: 10.1016/i.jneumeth.2007.03.005

[8] B. Rivet, A. Souloumiac, V. Attina, and G. Gibert, "Xdawn algorithm to enhance evoked potentials: Application to brain computer interface," IEEE Transactions on Biomedical Engineering, vol. 56, no. 8, pp. 2035-2043, 2009. doi: 10.1109/TBME.2009.2012869

[9] M. Kaper, P. Meinicke, U. Grossekathoefer, T. Lingner, and H. Ritter, "Bci competition 2003-data set iib: Support vector machines for the p300 speller paradigm," IEEE Transactions on Biomedical Engineering, vol. 51, no. 6, pp. 1073-1076, 2004. doi: 10.1109/TBME.2004.826698

[10] A. Rakotomamonjy and V. Guigue, "Bci competition iii: Dataset ii- ensemble of svms for bci p300 speller," IEEE Transactions on Biomedical Engineering, vol. 55, no. 3, pp. 1147-1154, 2008. doi: 10.1109/TBME.2008.915728

[11] V. N. Vapnik, Statistical learning theory New York: Berlin, Heidelberg: Springer, 1998.

[12] J. C. Burges, "A tutorial on support vector machines for pattern recognition," Data Mining and Knowledge Discovery vol. 2, no. 2, pp. 121-167, 1998. doi: $10.1023 / A: 1009715923555$
[13] A. L. Edsinger, "Robot manipulation in human environments," Ph. D. dissertation, Department of Electrical Engineering and Computer Science, Massachusetts Institute of Technology (MIT), Cambridge, Massachusetts. US, 2007.

[14] A. D. Luca, A. Albu-Schaffer, S. Haddadin, and G. Hirzinger, "Collision detection and safe reaction with the dlr-iii lightweight manipulator arm," in proceeding of IEEE International Conference On Intelligent Robots and Systems, Beijing, China, Oct. 9-15, 2006, pp. 1623-1630. doi: $10.1109 /$ IROS.2006.282053

[15] D. W. Robinson, "Design and analysis of series elasticity in closed-loop actuator force control," Ph. D. dissertation, Department of Mechanical Engineering, Massachusetts Institute of Technology (MIT), Cambridge, Massachusetts, US, 2000.

[16] M. Thulasidas, G. Cuntai, and W. Jiankang, "Robust classification of eeg signal for brain-computer interface," IEEE Transactions on Neural Systems and Rehabilitation Engineering, vol. 14, no. 1, pp. 24-29, 2006.

doi: 10.1109/TNSRE.2005.862695

[17] Y.-H. Liu, H.-P. Huang, and C.-H. Weng, "Recognition of electromyographic signals using cascaded kernel learning machine," IEEE/ASME Transactions on Mechatronics, vol. 12, no. 3, pp. 253-264, 2007. doi: 10.1109/TMECH.2007.897253

[18] N. V. Thakor, "Biopotentials and electrophysiology measurement," in The measurement. Instrumentation and sensors handbook, Boca Raton, Florida, US: CRC Pr I Llc, 1999, ch. 74.

[19] Y.-H. Liu, "Feature analysis and classifier design and their applications to pattern recognition and data mining," Ph.D. Dissertation, Department of Mechanical Engineering, National Taiwan University, Taipei, Taiwan, 2003.

[20] D. Zhang and G. Lu, "Generic fourier descriptor for shape-based image retrieval," in proceeding of IEEE International Conference on Multimedia and Expo, Lausanne, Switzerland, Aug. 26-19, 2002, vol. 1, pp. 425-428. doi: $10.1109 /$ ICME.2002.1035809 